\title{
Genomic imprinting
}

National Cancer Institute ( $\mathrm{NCl})$

\section{Source}

National Cancer Institute (NCI). Genomic Imprinting.

An epigenetic process resulting in the inactivation of an allele depending on which parent it was inherited from. Genomic imprinting can have clinical relevance because it may affect the expression of a gene mutation (i.e., the phenotype) in the offspring of an affected parent depending on which parent is passing on the mutation. 